\title{
Multiplex Human Malaria Array: Quantifying Antigens for Malaria Rapid Diagnostics
}

\author{
Ihn Kyung Jang, ${ }^{1 \star}$ Abby Tyler, ${ }^{2}$ Chris Lyman, ${ }^{2}$ John C. Rek, ${ }^{3}$ Emmanuel Arinaitwe, ${ }^{3}$ Harriet Adrama, ${ }^{3}$ Maxwell Murphy, ${ }^{4}$ \\ Mallika Imwong, ${ }^{5}$ Stephane Proux, ${ }^{6}$ Warat Haohankhunnatham, ${ }^{6}$ Rebecca Barney, ${ }_{1}^{1}$ Andrew Rashid, ${ }^{1}$ Michael Kalnoky, ${ }^{1}$ \\ Maria Kahn, ${ }^{1}$ Allison Golden, ${ }^{1}$ François Nosten, ${ }^{6,7}$ Bryan Greenhouse, ${ }^{4}$ Dionicia Gamboa, ${ }^{8}$ and Gonzalo J. Domingo ${ }^{1}$ \\ ${ }^{1}$ Diagnostics, PATH, Seattle, Washington; ${ }^{2}$ Quansys Biosciences, Logan, Utah; ${ }^{3}$ Infectious Diseases Research Collaboration, Kampala, Uganda; \\ ${ }^{4}$ Department of Medicine, University of California at San Francisco, San Francisco, California; ${ }^{5}$ Faculty of Tropical Medicine, Department of \\ Molecular Tropical Medicine and Genetics, Mahidol University, Bangkok, Thailand; ${ }^{6}$ Faculty of Tropical Medicine, Mahidol-Oxford Tropical \\ Medicine Research Unit, Shoklo Malaria Research Unit, Mahidol University, Mae Sot, Thailand; ${ }^{7}$ Nuffield Department of Medicine, Centre for \\ Tropical Medicine and Global Health, University of Oxford, Oxford, United Kingdom; ${ }^{8}$ Department of Cellular and Molecular Sciences, Institute of \\ Tropical Medicine Alexander von Humboldt, Universidad Peruana Cayetano Heredia, Lima, Peru
}

\begin{abstract}
Malaria antigen detection through rapid diagnostic tests (RDTs) is widely used to diagnose malaria and estimate prevalence. To support more sensitive next-generation RDT development and screen asymptomatic malaria, we developed and evaluated the Q-Plex ${ }^{\mathrm{TM}}$ Human Malaria Array (Quansys Biosciences, Logan, UT), which quantifies the antigens commonly used in RDTs-Plasmodium falciparum-specific histidine-rich protein 2 (HRP2), P. falciparumspecific lactate dehydrogenase (Pf LDH), Plasmodium vivax-specific LDH $(P v$ LDH), and Pan malaria lactate dehydrogenase (Pan LDH), and human C-reactive protein (CRP), a biomarker of severity in malaria. At threshold levels yielding $99.5 \%$ or more diagnostic specificity, diagnostic sensitivities against polymerase chain reaction-confirmed malaria for HRP2, Pf LDH, PV LDH, and Pan LDH were 92.7\%, 71.5\%, 46.1\%, and 83.8\%, respectively. P. falciparum culture strains and samples from Peru indicated that HRP2 and $P f$ LDH combined improves detection of $P$. falciparum parasites with $h r p 2$ and $h r p 3$ deletions. This array can be used for antigen-based malaria screening and detecting $h r p 2 / 3$ deletion mutants of $P$. falciparum.
\end{abstract}

\section{INTRODUCTION}

There are more than 200 million cases of human malaria worldwide, with some countries in the control phase of malaria and others seeking to eliminate malaria. ${ }^{1}$ Malaria diagnosis and treatment is mostly through detection of the parasite by microscopy or parasite antigens through rapid diagnostic tests (RDTs). ${ }^{2}$ Many commonly used RDTs target two malaria antigens: histidine-rich protein (HRP2) and the essential enzyme, Plasmodium LDH (pLDH). ${ }^{3}$ Histidinerich protein 2, a Plasmodium falciparum-specific protein, and its homolog HRP3 are used as biomarkers to confirm $P$. falciparum infection. Unfortunately, RDTs recognizing the common epitopes found in HRP2 and HRP3 result in failed diagnosis for clinical $P$. falciparum infections because of partial or complete deletions of $h r p 2$ and $h r p 3$, and consequently no expression of proteins. ${ }^{4}$ Characterization of $h r p 2 / 3$ deletions by polymerase chain reaction (PCR) is time consuming and expensive for larger scale clinical studies. ${ }^{4}$ Antibodies targeting a conserved epitope in pLDH can detect all human Plasmodium species Pan malaria lactate dehydrogenase (Pan LDH). pLDH can also be used for species-specific detection for $P$. falciparum and $P$. vivax infection using antibodies that recognize species-specific epitopes in $P$. falciparum-specific lactate dehydrogenase ( $P f L D H)$ and $P$. vivax-specific LDH $(P v L D H) .{ }^{5,6}$

The performance of RDTs is driven by the underlying relationship between antigen concentration and parasite density. This relationship is defined by multiple factors including antigen turnover and decay rates, affinity characteristics of the antigen capture reagents, whether infections are recent or asymptomatic, and probably also by host factors such as previous exposure to malaria. ${ }^{7-10}$ Two quantitative multiplex tests, bead-based assay and chemiluminescence-based

*Address correspondence to Ihn Kyung Jang, Diagnostics, PATH, 2201 Westlake Ave., Suite 200, Seattle, WA 98121. E-mail: ikjang@ path.org
ELISA, have been described. ${ }^{11,12}$ The bead-based assay detects Pan aldolase, Pan LDH, and HRP2, whereas the ELISA detects HRP2, $P v$ LDH, Pan LDH, and C-reactive protein (CRP). An expanded version of this ELISA including an assay for $P f \mathrm{LDH}$ was developed for research use and is now commercially available as the Q-Plex ${ }^{\mathrm{TM}}$ Human Malaria Array (5-Plex [Quansys Bioscience, Logan, UT]). In this study, the diagnostic performance of this new test was assessed.

\section{METHODS}

Ethics and source of specimen. Clinical samples from asymptomatic infections in Myanmar and Uganda were collected under informed consent and with institutional review board (IRB) approvals, as described previously. ${ }^{13}$ A randomly selected subset of samples was used in this study. Seventy-five clinical samples with $P$. falciparum or $P$. vivax infections from symptomatic infections in Vietnam, which were also collected under informed consent and IRB approval, were purchased from Discovery Life Sciences (Los Osos, CA). Twenty-six $P$. falciparum clinical samples were obtained with informed consent and with IRB approval from Universidad Peruana Cayetano Heredia (UPCH, Lima, Peru) (UPCH 52707). Plasmodium malariae- and Plasmodium ovale-infected blood samples were obtained from the Foundation for Innovative New Diagnostics (FIND, Geneva, Switzerland) Specimen Bank.

Five $P$. falciparum laboratory strains were used in this study, W2, D10, Dd2, and HB3 (BEI Resources, Manassas, VA), and 3BD5 (the National Institute of Allergy and Infectious Diseases, Bethesda, MD). The wild-type strain W2 is $h r p 2^{+} / h r p 3^{+}$, D10 and Dd2 are $h r p 2^{-} / h r p 3^{+}, \mathrm{HB} 3$ is $h r p 2^{+} / h r p 3^{-}$, and $3 \mathrm{BD} 5$ is $h r p 2^{-} /$ hrp3 ${ }^{-}$. All strains were cultured and harvested at the ring stage (> 99\%) after synchronization, as described previously. ${ }^{12}$ The parasite number was determined by microscopy. Parasite-infected red blood cell pellets were used to generate a dilution series derived from a pool of uninfected blood (see Supplemental Methods). 
Laboratory tests for HRP2/pLDH and parasite DNA. Quantification of malaria antigens and CRP was performed using the 5-Plex (Quansys Bioscience) (see Supplemental Methods). The speciation of all field samples except those from Vietnam was confirmed by quantitative PCR methods. ${ }^{13}$ Photo-induced electron transfer real-time PCR was used to characterize samples from Vietnam (see Supplemental Methods). The samples from Peru were previously characterized for the presence or absence of $h r p 2$ and $h r p 3$ by PCR and confirmed for HRP proteins by conventional ELISA. ${ }^{14}$

Statistical analysis. All statistical analyses were performed using GraphPad Prism, version 6.0 (GraphPad, San Diego, CA). The detail in data analysis is described in Supplemental Methods.

\section{RESULTS}

Performance of 5-plex array. The analytical performance of the 5-Plex was evaluated based on antigen quantification as determined from the pixel intensity on the array spot relative to a standard curve (Supplemental Table 1 and Supplemental Table 2). The 5-Plex was challenged with a validation sample set consisting of 462 blood samples from PCR-confirmed positive and negative cases with $P$. falciparum and $P$. vivax infection from Uganda, Myanmar, and Vietnam. All four malaria biomarkers, HRP2, Pan LDH, Pf LDH, and $P v \mathrm{LDH}$, found in significantly higher amounts in the respective malaria-positive samples are compared with the negative control group $(P<$ 0.0001) (Figure 1, Supplemental Table 3). Receiver operating characteristic $(\mathrm{ROC})$ analysis was conducted, and the area under the ROC curves (AUCs) with 95\% Cls for each biomarker was calculated in relation to the discrimination between malaria cases and controls. The AUCs were 0.979 (95\% Cl: 0.960-0.998) for HRP2, 0.939 (95\% Cl: 0.911-0.968) for Pf LDH, 0.926 (95\% Cl: 0.898-0.954) for Pv LDH, and 0.948 (95\% Cl: 0.928-0.968) for Pan LDH (Supplemental Figure 1).

Optimal cutoff points were calculated targeting a specificity of $\geq 99.5 \%$ (Table 1) against PCR-confirmed negative samples
A
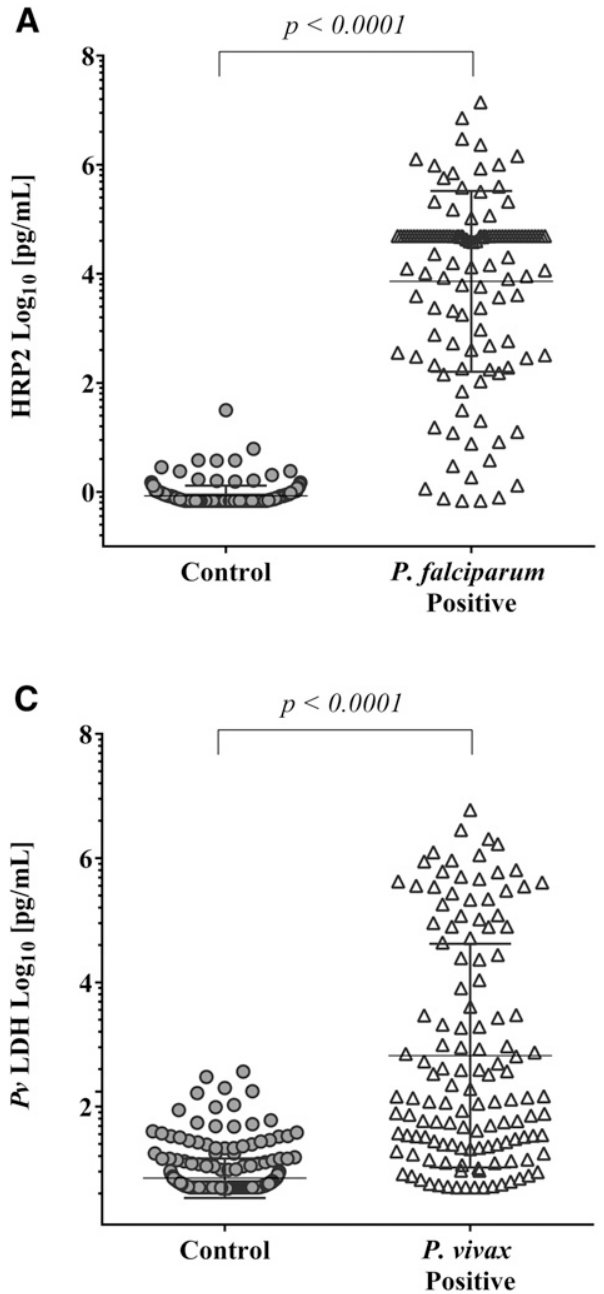

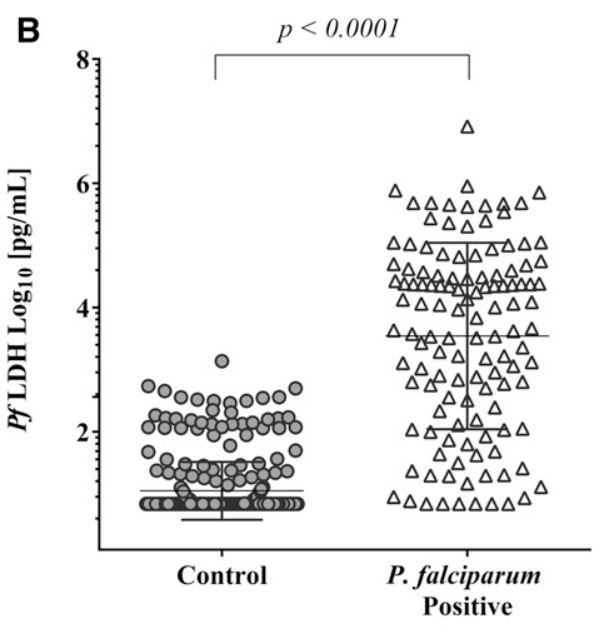

D

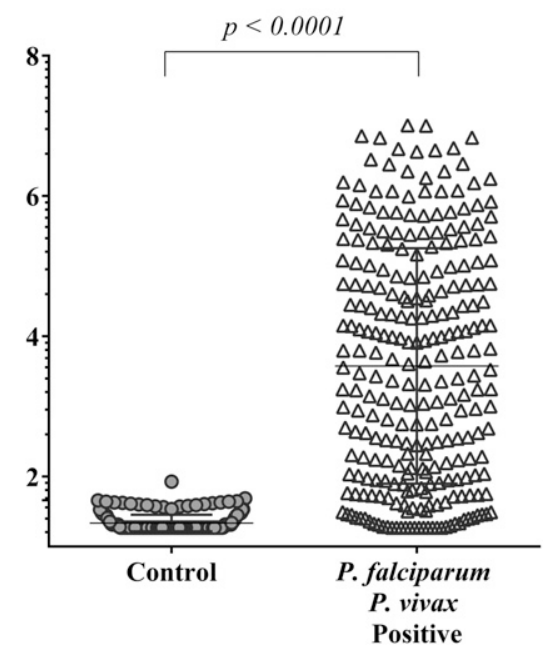

FIGURE 1. Dot plots of the concentration for each biomarker as determined on the 5-Plex infection-negative and infection-positive samples (as summarized in Supplemental Table 2). (A) Histidine-rich protein 2 (HRP2), (B) Plasmodium falciparum-specific lactate dehydrogenase (Pf LDH), (C) $P$. vivax-specific LDH $(P v L D H)$, and (D) Pan malaria lactate dehydrogenase (Pan LDH) assays. Malaria-negative samples (no parasites, $n=196)$ from Uganda and Vietnam were used as controls for the HRP2 and Pan LDH assays, whereas samples that do not have the corresponding Plasmodium species (non- $P$. falciparum malaria, $n=128$; non- $P$. vivax malaria, $n=123$ ) were used as controls for $P v$ LDH and $P f$ LDH assays to address the falsepositive results from cross-reactivity of pLDH. The longer horizontal line in each blot indicates the mean, and the upper and lower shorter lines indicate one standard deviation (SD). The Mann-Whitney U-test was used to calculate $P$-values; $P<0.05$ was considered statistically significant. 
TABLE 1

Performance characteristics for each malaria antigen assay used in the 5-Plex HRP2, Pf LDH for $P$. falciparum, $P v$ LDH for $P$. vivax, and Pan LDH for all malaria species

\begin{tabular}{|c|c|c|c|c|}
\hline Analyte & $\begin{array}{l}\text { Target polymerase chain } \\
\text { reaction-confirmed infection }\end{array}$ & Threshold $(\mathrm{pg} / \mathrm{mL})$ & Sensitivity $(95 \% \mathrm{Cl})$ & Specificity $(95 \% \mathrm{Cl})$ \\
\hline HRP2 & P. falciparum & 6.9 & $92.7(86.6-96.6)$ & $99.5(97.2-100)$ \\
\hline$P f \mathrm{LDH}$ & P. falciparum & 553.6 & $71.5(62.7-79.3)$ & 99.7 (98.3-100) \\
\hline Pv LDH & P. vivax & 314.8 & $46.1(37.3-55.1)$ & 99.7 (98.3-100) \\
\hline Pan LDH & All malaria & 49.0 & 83.8 (78.9-88.1) & $99.5(97.2-100.0)$ \\
\hline
\end{tabular}

or non-cognate Plasmodium subspecies infection. From analyzing the validation sample set described in Figure 1, the HRP2 assay displayed the best diagnostic performance characteristics with a sensitivity and specificity of $92.7 \%$ and $99.5 \%$, respectively. Analysis with 15 validation samples with dual infection of $P$. falciparum and $P$. vivax revealed that the 5-Plex identified seven samples as coinfected, with the remaining eight samples being only $P$. vivax positive because of very low $P$. falciparum parasitemia in the range of $1-40 \mathrm{p} / \mu \mathrm{L}$.

A dilution series of five $P$. falciparum strains in the range of 66.7-0.3 p/ $\mu \mathrm{L}$ with variable $h r p 2$ and $h r p 3$ genotypes were also analyzed on the 5-Plex. The HRP2 assay detected parasites from two strains, the wild-type W2 $\left(h r p 2^{+} / h r p 3^{+}\right)$and the HB3 $\left(h r p 2^{+} / h_{r p 3}{ }^{-}\right)$, to as few as $0.8 \mathrm{p} / \mu \mathrm{L}$ and $0.3 \mathrm{p} / \mu \mathrm{L}$, respectively. In parallel, the $P f \mathrm{LDH}$ assay detected parasites at $66.7 \mathrm{p} / \mu \mathrm{L}$ and $22.2 \mathrm{p} / \mu \mathrm{L}$, respectively (Supplemental Figure 2). Screening of $h r p$ mutants with the $P f$ LDH assay using hrp2 deletion strains D10 and Dd2 and 3BD5, a hrp2/3 double mutant detected parasitemia of $7.4 \mathrm{p} / \mu \mathrm{L}, 22.2 \mathrm{p} / \mu \mathrm{L}$, and 66.7 $\mathrm{p} / \mu \mathrm{L}$, respectively.

The 5-Plex was also evaluated with a panel of 26 parasite count-adjusted samples derived from clinical samples collected in Peru and previously characterized for $h r p 2 / 3$ deletions by PCR. ${ }^{14}$ All 26 samples were accurately identified as $P$. falciparum by the 5-Plex by detecting either HRP2 or Pf LDH. A subset of nine from 12 (75\%) samples with hrp2/3 deletion in this panel was correctly identified as HRP negative with the 5-Plex HRP2 assay (Table 2).

Cross-reactivity with other Plasmodium species. The 5Plex was further investigated with $17 P$. malariae and eight $P$. ovale PCR-confirmed samples. Of the $17 \mathrm{P}$. malariaepositive samples, 14/17 (82.4\%) were positive by the Pan LDH assay and only $5 / 17$ (29.4\%) were positive by the $P f$ LDH assay (Supplemental Figure 3). The $P$. malariae samples had relatively high parasite densities in the range of 75.2-988.3 $\mathrm{p} / \mathrm{\mu L}$. Of the eight PCR P. ovale-positive samples, 3/8 (37.5\%) were positive by Pan LDH assay, with parasite densities in the range of 0.1-8.0 p/ $\mu \mathrm{L}$. None (0/8) was positive by $\mathrm{Pf} L \mathrm{LH}$ assay. The
$P$. malariae and $P$. ovale samples were negative with the HRP2 and $P v$ LDH assays.

\section{DISCUSSION}

The 5-Plex is able to differentiate $P$. falciparum infections, including those with $h r p 2 / 3$ deletions, $P$. vivax infection, and also detect $P$. ovale and $P$. malariae. Similar multiplex assays have been developed on the Luminex bead-based platform but are not available as commercial kits. ${ }^{11,15,16}$

In contrast to the previously described 4-Plex array, ${ }^{12}$ the performance of the new 5-Plex is significantly improved: the prozone effect in the HRP2 assay is mitigated along with the wider dynamic range (Supplemental Figure 4), ${ }^{17}$ and through the Pf LDH, it can identify samples with suspected hrp2/3 deletions.

At a specificity of $>99.5 \%$, the sensitivity for detecting any species of malaria using the Pan LDH assay was $83.8 \%$ and for $P$. falciparum infection using HRP2 was $92.7 \%$. When identifying $P$. falciparum and $P$. vivax infections through the $P f$ $\mathrm{LDH}$ and $P v$ LDH assays, the sensitivity was reduced to $71.5 \%$ and $46.1 \%$, respectively. The Pf LDH assay does show crossreactivity with $P$. malariae. The sequence similarity of the related proteins from $P$. malariae and $P$. falciparum may contribute to cross-reactivity. In addition, elevated levels of CRP, an acute phase inflammatory protein, were consistently observed in febrile malaria-positive individuals, in contrast to asymptomatic malaria-positive individuals (Supplemental Figure 5), supporting the correlation of the CRP level with complications in malaria. ${ }^{18,19}$

The data presented in this study suggest that the Q-Plex ${ }^{\mathrm{TM}}$ Human Malaria Array can be used on whole blood samples to operate high throughput screening tool for malaria surveillance including the identification of $h r p 2 / 3-$ deleted $P$. falciparum before resorting to more complex molecular tests for the gene deletions. ${ }^{4}$ Although HRP2 persists after malaria clearance, the pLDH level rapidly declines on treatment. ${ }^{20}$ The capability of the array to simultaneously quantify these malaria antigens may

TABLE 2

Performance of the 5-Plex to identify $P$. falciparum using Pf LDH and Pan LDH assays with hrp2/3-deleted $P$. falciparum samples

\begin{tabular}{|c|c|c|c|c|c|c|c|}
\hline \multirow[b]{2}{*}{ Category } & \multirow{2}{*}{$\frac{h r p 2 / 3 \text { deletion by polymerase chain reaction }}{\text { Sample number }}$} & \multicolumn{4}{|c|}{ 5-Plex, positive sample number. } & \multicolumn{2}{|c|}{$\%$ Accuracy } \\
\hline & & HRP2 & $P f \mathrm{LDH}$ & $P v$ LDH & Pan LDH & Pf classification & hrp2/3 deletion \\
\hline hrp2+/hrp3+ & 5 & 5 & 5 & 0 & 5 & 100 & NA \\
\hline hrp2+/hrp3- & 6 & 6 & 6 & 0 & 6 & 100 & NA \\
\hline hrp2-/hrp3+ & 3 & $1^{*}$ & 3 & 0 & 3 & 100 & NA \\
\hline hrp2-/hrp3- & 12 & $3+$ & 12 & 0 & 12 & 100 & 75.0 \\
\hline
\end{tabular}

$\mathrm{HRP}=$ histidine-rich protein; $\mathrm{NA}=$ not applicable; $P$. falciparum = Plasmodium falciparum; $P f \mathrm{LDH}=P$. falciparum-specific lactate dehydrogenase; Pan $\mathrm{LDH}=\mathrm{Pan}$ malaria lactate dehydrogenase $P v \mathrm{LDH}=P$. vivax-specific LDH

* $230 \mathrm{ng} / \mathrm{mL}$ of HRP2 by the 5-Plex; no conventional ELISA data available.

† One sample was detected for $40,000 \mathrm{pg} / \mathrm{mL}$ of HRP2 by the 5 -Plex but was not quantifiable by the conventional ELISA. Two other samples were 18.9 and $9 \mathrm{pg} / \mathrm{mL}$ of HRP2 by the 5 -Plex; no conventional ELISA data available. 
be of significant value to the future studies that could provide deep insight into the malaria infection status classification as well as the speciation of suspected malaria.

Received October 14, 2019. Accepted for publication January 13, 2020.

Published online March 16, 2020.

Note: Supplemental file appears at www.ajtmh.org.

Acknowledgments: We greatly acknowledge the support of human volunteers who willingly donated their blood samples for this study. We thank Sophie Knudson and Teri Scott for their assistance in manuscript proofing and editing.We are grateful to FIND Specimen Bank for the human biological biobank samples and David Boyle for critical review of this manuscript.

Financial support: This work was supported by the Bill \& Melinda Gates Foundation (Grant no. OPP1135840).

Disclaimer: The findings or conclusions in this study are those of the authors and do not necessarily represent the official position of the Bill \& Melinda Gates Foundation.

Disclosure: A. T. and C. L. are employed by Quansys Biosciences (Logan, Utah), the manufacturer of the 5-Plex.

Authors' addresses: Ihn Kyung Jang, Rebecca Barney, Andrew Rashid, Michael Kalnoky, Maria Kahn, Allison Golden, and Gonzalo Jose Domingo, Diagnostics, PATH, Seattle, WA, E-mails: ikjang@path.org, rbarney@path.org, arashid@path.org, mkalnoky@gmail.com, mkahn@ path.org, and gdomingo@path.org. Abby Tyler and Chris Lyman, Quansys Biosciences, Research and Development, Logan, UT, E-mails: atyler@quansysbio.com and clyman@quansysbio.com. John C. Rek, Emmanuel Arinaitwe, and Harriet Adrama, Infectious Diseases Research Collaboration, Kampala, Uganda, E-mails: jrek@idrc-uganda.org, earinaitwe@idrc-uganda.org, and harriet.adrama@gmail.com. Maxwell Murphy and Bryan Greenhouse, Department of Medicine, University of California San Francisco, San Francisco, CA, E-mails: maxwell.murphy@ ucsf.edu and bryan.greenhouse@ucsf.edu. Mallika Imwong, Faculty of Tropical Medicine, Department of Molecular Tropical Medicine and Genetics, Mahidol University, Bangkok, Thailand, E-mail: mallika.imw@ mahidol.ac.th. Stephane Proux and Warat Haohankhunnatham, Faculty of Tropical Medicine, Mahidol-Oxford Tropical Medicine Research Unit, Shoklo Malaria Research Unit, Mahidol University, Mae Sot, Thailand, E-mails: steph@shoklo-unit.com and warat@shoklo-unit.com. Francois Nosten, Faculty of Tropical Medicine, Mahidol-Oxford Tropical Medicine Research Unit, Shoklo Malaria Research Unit, Mahidol University, Mae Sot, Thailand, and Nuffield Department of Medicine, Centre for Tropical Medicine and Global Health, University of Oxford, Oxford, United Kingdom, E-mail: francois@tropmedres.ac. Dionicia Gamboa, Department of Cellular and Molecular Sciences, Institute of Tropical Medicine Alexander von Humboldt, Universidad Peruana Cayetano Heredia, Lima, Peru, E-mail: dionigamboa@yahoo.com.

This is an open-access article distributed under the terms of the Creative Commons Attribution (CC-BY) License, which permits unrestricted use, distribution, and reproduction in any medium, provided the original author and source are credited.

\section{REFERENCES}

1. WHO, 2018. World Malaria Report 2018. Geneva, Switzerland: World Health Organization.

2. Wongsrichanalai C, Barcus MJ, Muth $S$, Sutamihardja A, Wernsdorfer $\mathrm{WH}, 2007$. A review of malaria diagnostic tools: microscopy and rapid diagnostic test (RDT). Am J Trop Med Hyg 77: 119-127.

3. Mouatcho JC, Goldring JP, 2013. Malaria rapid diagnostic tests: challenges and prospects. J Med Microbiol 62: 1491-1505.

4. Cheng Q, Gatton ML, Barnwell J, Chiodini P, McCarthy J, Bell D, Cunningham J, 2014. Plasmodium falciparum parasites lacking histidine-rich protein 2 and 3: a review and recommendations for accurate reporting. Malar J 13: 283.

5. Hurdayal R, Achilonu I, Choveaux D, Coetzer TH, Dean Goldring JP, 2010. Anti-peptide antibodies differentiate between plasmodial lactate dehydrogenases. Peptides 31: 525-532.

6. Piper RC, Buchanan I, Choi YH, Makler MT, 2011. Opportunities for improving pLDH-based malaria diagnostic tests. Malar J 10: 213.

7. Ho MF et al., 2014. Circulating antibodies against Plasmodium falciparum histidine-rich proteins 2 interfere with antigen detection by rapid diagnostic tests. Malar J 13: 480.

8. Markwalter CF, Jang IK, Burton RA, Domingo GJ, Wright DW, 2017. Biolayer interferometry predicts ELISA performance of monoclonal antibody pairs for Plasmodium falciparum histidine-rich protein 2. Anal Biochem 534: 10-13.

9. Dalrymple U, Arambepola R, Gething PW, Cameron E, 2018. How long do rapid diagnostic tests remain positive after anti-malarial treatment? Malar J 17: 228.

10. Ranadive $\mathrm{N}$ et al., 2017. Limitations of rapid diagnostic testing in patients with suspected malaria: a diagnostic accuracy evaluation from Swaziland, a low-endemicity country aiming for malaria elimination. Clin Infect Dis 64: 1221-1227.

11. Rogier E et al., 2017. Bead-based immunoassay allows subpicogram detection of histidine-rich protein 2 from Plasmodium falciparum and estimates reliability of malaria rapid diagnostic tests. PLoS One 12: e0172139.

12. Jang IK et al., 2019. Simultaneous quantification of Plasmodium antigens and host factor $C$-reactive protein in asymptomatic individuals with confirmed malaria by use of a novel multiplex immunoassay. J Clin Microbiol 57: e00948-18.

13. Das $S$ et al., 2017. Performance of a high-sensitivity rapid diagnostic test for Plasmodium falciparum malaria in asymptomatic individuals from Uganda and Myanmar and naive human challenge infections. Am J Trop Med Hyg 97: 1540-1550.

14. Gamboa D et al., 2010. A large proportion of $P$. falciparum isolates in the Amazon region of Peru lack pfhrp2 and pfhrp3: implications for malaria rapid diagnostic tests. PLoS One 5: e8091.

15. Plucinski MM et al., 2019. Screening for pfhrp2/3-deleted Plasmodium falciparum, non-falciparum, and low-density malaria infections by a multiplex antigen assay. J Infect Dis 219: 437-447.

16. Plucinski MM, Rogier E, Dimbu PR, Fortes F, Halsey ES, Aidoo M, 2017. Estimating the added utility of highly sensitive histidinerich protein 2 detection in outpatient clinics in sub-saharan africa. Am J Trop Med Hyg 97: 1159-1162.

17. Luchavez J, Baker J, Alcantara S, Belizario V Jr., Cheng Q, McCarthy JS, Bell D, 2011. Laboratory demonstration of a prozone-like effect in HRP2-detecting malaria rapid diagnostic tests: implications for clinical management. Malar J 10: 286

18. Gillespie SH, Dow C, Raynes JG, Behrens RH, Chiodini PL, McAdam KP, 1991. Measurement of acute phase proteins for assessing severity of Plasmodium falciparum malaria. J Clin Pathol 44: 228-231.

19. Andrade BB, Reis-Filho A, Souza-Neto SM, Clarencio J, Camargo LM, Barral A, Barral-Netto M, 2010. Severe Plasmodium vivax malaria exhibits marked inflammatory imbalance. Malar J 9: 13.

20. Iqbal J, Siddique A, Jameel M, Hira PR, 2004. Persistent histidinerich protein 2 , parasite lactate dehydrogenase, and panmalarial antigen reactivity after clearance of Plasmodium falciparum monoinfection. J Clin Microbiol 42: 4237-4241. 